\title{
Effects of Low-to-medium Intensity Intradialytic Resistance Training in Elderly Chronic Hemodialysis Patients: Preliminary Effects after One Year of Intervention
}

\author{
Kotomi Shiota ${ }^{1,2}$ \\ ${ }^{1}$ Organization for University Research Initiatives, Waseda University \\ ${ }^{2}$ Institute of Health and Community Sports \\ Floor 3, Bldg. No. 9 Waseda Campus, Waseda University, 1-6-1 Nishiwaseda, Shinjuku-ku, Tokyo, 169-8050 JAPAN \\ Kotomi Shiota (shiota@aoni.waseda.jp) \\ [Received August 11, 2016; Accepted June 7, 2017; Published online July 7, 2017]
}

\begin{abstract}
Objective: To analyze the effects of intradialytic resistance training on physical function in 8 hemodialysis patients aged $\geq 60$ years. Method: Physical function, blood pressure, heart rate, and biochemical data were analyzed before and after 3 and 12 months of exercise therapy. Focusing on muscle strengthening of the trunk and legs while on bed rest, the exercise load of the intervention was individualized for each patient. Results: Significant differences were observed in ankle plantar flexor strength, the Timed Up and Go test, and maximum walking speed between before and after 3 months of intervention. However, no significant differences were observed from 3 to 12 months, indicating that there was no long-term improvement upon lowto-medium resistance training during hemodialysis. Conclusion: Further studies with increased sample sizes are required to elucidate the appropriate intensity, frequency, and duration for an effective resistance training program in this population.
\end{abstract}

Keywords: physical function, activities of daily living, training program

\section{Introduction}

In recent years, the number of hemodialysis (HD) patients has greatly increased, especially in terms of elderly patients and patients with over 10 years of HD history. HD patients frequently experience skeletal muscle weakening or atrophy, reduced muscle blood flow distribution, reduced cardiopulmonary function, and decreases in exercise tolerance (Longenecker et al., 2002). In particular, previous studies have shown that HD patients without a regular exercise habit show even greater decreases in these functions, as well as higher mortality (O'Hare et al., 2003; Pechter et al., 2014). Consequently, these decreases in physical function have been reported to lead to decreases in the activities of daily living (ADL) and quality of life (QOL) of the patients, thus forming a vicious cycle (O'Hare et al., 2003).

Additionally, many HD patients have diabetes or other lifestyle-related diseases as the underlying disease, and often suffer from multiple diseases, ranging from internal organ failure to joint disease. In elderly HD patients, complications such as heart failure and hypotension commonly occur, resulting in further lowering of the QOL. In turn, their decreased physical function forces them to rest even more, thereby lowering their exercise tolerance and resulting in disuse syndrome, indicating that there is a need for countermeasures to improve HD patients' QOL and to prevent or mitigate disuse syndrome.

In recent years, exercise intervention for HD patients has been recommended as one such countermeasure, and its reported effects include improved exercise tolerance and cardiopulmonary function (Heiwe and Jacobson 2014; Johansen and Painter, 2012; Bennett et al., 2010; Kouidi 2002; Deligiannis et al., 1999; Vilsteren et al., 2005). However, many of these previous studies assessed 
the intervention effect of intradialytic aerobic exercise during a relatively short period of time, with a high load, and in subjects with an average age in the $40 \mathrm{~s}$ and $50 \mathrm{~s}$ (Deligiannis et al., 1999; Vilsteren et al., 2005). In contrast, in elderly HD patients, the appropriate aerobic exercise intervention remains unknown and these patients may be poor candidates for such interventions, as it may be difficult to continuously perform the exercise. On the other hand, one useful characteristic of resistance training (RT) is that, among the physical dysfunctions in elderly HD patients, the legs show an especially severe decrease in muscle strength levels, equivalent to 40-70 $\%$ of that of healthy subjects (Shiota et al., 2009); generally, leg muscle strength has been found to show a high correlation with many items of physical functioning (e.g. walking speed), which in turn are strongly associated with autonomy and independence in ADL (Johansen, et al., 2003). Therefore, it is important to take a localized approach to enhance the muscle groups needed for ADL in this patient population. Moreover, previous studies investigating the effects of RT in chronic renal failure patients showed that RT-only exercise intervention was equally effective to aerobic exercise, both when RTonly was compared with aerobic exercise-only and a combination of RT and aerobic exercise (Snowling and Hopkins, 2006; Moinuddin and Leehey, 2008; Reid et al., 2010).

However, there is very little prior research on the appropriate RT methods for elderly patients undergoing HD. The intensity, frequency, and amounts of exercises that are suitable for elderly HD patients need to be investigated, in addition to their effects, to establish the role of RT in this patient population. Therefore, the present study aimed to clarify the changes in physical function (primary outcome), as well as the effects on biochemical and blood pressure data (secondary outcomes), after 1 year of performing low-to-medium intensity RT during HD in elderly HD patients.

\section{Method}

\subsection{Subjects}

This study was approved by the Ethics Committee on Human Research of Ryotokuji University. The protocol for the research project conforms to the provisions of the Declaration of Helsinki (as revised in Tokyo in 2004). The researcher described
Table 1 Inclusion and exclusion criteria.

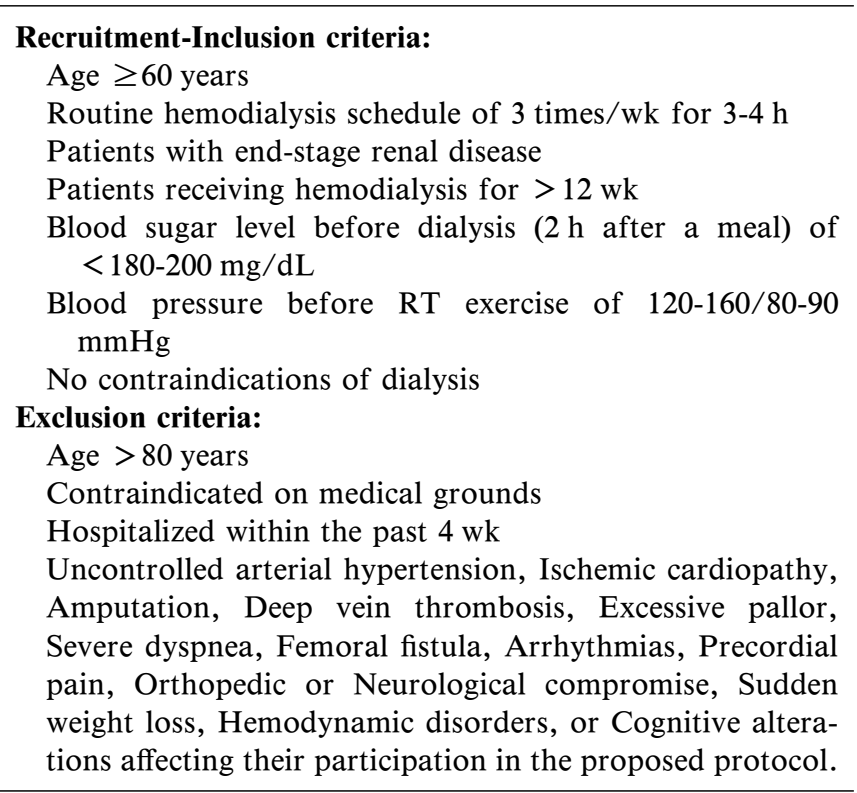

Abbreviations: RT, Resistance Training

the research purposes, methods, risks, and benefits to the subjects before obtaining written informed consent for participation in the study.

The final study population comprised 8 HD patients who met the selection criteria outlined in Table 1. In this study, we defined 'elderly HD patient' as those over 60 years old, and those who provided consent to participate in this experiment, from among outpatients visiting a clinic specializing in HD treatment in Tokyo.

\subsection{Measurements}

The physical function measurement items, representing the main outcome of the present study, were measured prior to RT intervention (baseline period [BS]), and 3 months ( $3 \mathrm{M}$ ) and 12 months after (12 M) intervention initiation, with all measurements being taken $2 \mathrm{~h}$ before HD. During the physical function measurements, the grip strength and leg muscle strength were measured for the muscle strength assessments. Grip strength was measured using a grip strength dynamometer (Takei, Tokyo, Japan; Scientific Instruments, Japan); the left and right sides were measured twice each, and the maximum values were used for the analysis. Leg muscle strength was measured with a simplified myodynamometer ( $\mu$-tas F-1; Anima), assessing knee extension, ankle dorsiflexion, and plantar flexion. 
For assessing the hip flexor, knee extensor, and ankle dorsiflexor strengths, the patients were seated in a chair with $90^{\circ}$ hip and knee flexion, and an attachment was placed on the distal femur, the distal lower leg, and the dorsum of the foot, respectively; subsequently, a maximum contraction of the target muscle was maintained for 5 seconds and measured. During the ankle plantar flexor strength test, a manual myodynamometer was attached to the plantar region and measurements were taken from a position of $0^{\circ}$ ankle plantar dorsiflexion while sitting. Measurements for all muscles were obtained with a discretionary level of contraction lasting 5 seconds; all measurements were taken twice, with 2 min of rest between each, and the maximum value was used. Further, body swaying over 30 seconds of static standing was measured (G-620; Anima) by two tests (eyes open and eyes closed) as a balance ability assessment. Finally, normal and maximum $10-\mathrm{m}$ walking speeds were measured, as well as a Timed Up and Go test (TUG), for assessing movement function. The $10 \mathrm{~m}$ of walking was preceded and followed by $3-\mathrm{m}$ approach intervals. The walking speed and number of steps were measured based on the foot in the swing phase, starting from the starting line of the 10-m measurement interval, and the measurement was concluded when the foot in the swing phase passed the goal line. The normal and maximum pace speeds were each measured two times, and the maximum values were used. During the TUG (Podsiadlo and Richardson, 1991), the subjects stood from a seated position in a chair, and changed direction at the position of a target $3 \mathrm{~m}$ ahead, and then returned and sat back down in the chair; the time required to complete the distance was measured. The walking was performed at a quick pace, but not fast enough to be considered running. The procedure was implemented twice and the maximum value was used.

As secondary outcomes, blood pressure and heart rate data, measured automatically during the intervention, along with biochemical data, were collected from the medical records after 1 year of RT. For the blood pressure data, data on the systolic blood pressure (SBP) and diastolic blood pressure (DBP) before and after HD were acquired. Subsequently, data from the year before RT intervention were employed as the Base Line (BS), and the 12-month means for each item were calculated for the onceweekly RT implementation day (implementation day) and the remaining twice-weekly RT non-implementation days (non-implementation days) out of the 3 weekly HD days. For the biochemical data, data from the year before RT intervention were used as the BS period; hematocrit $(\mathrm{Ht})$ and hemoglobin $(\mathrm{Hb})$ data before and after HD were collected every month, and data for albumin (Alb) were collected once every 3 months, after which the 12-month means were calculated.

\subsection{Intervention}

The RT intervention was timed no sooner than 30 min and no later than $2 \mathrm{~h}$ after the start of HD with stable hemodynamics. The intervention started when the patients' systolic blood pressure was under $150 \mathrm{mmHg}$. Additionally, patients who needed to take medicine for their blood pressure continued doing so depending on their hemodynamic stability before the intervention. RT was performed once a week using resistance elastic bands and tubing and exercise balls, primarily focusing on trunk and leg muscle strengthening: leg abduction, leg adduction, plantar flexion, dorsiflexion, straight-leg raise, bentknee raise, knee extension, knee flexion, sit-up, back-bridge (hip-up), under supervision by a physical therapist who served as a personal trainer. The exercise load was set on an individual basis, intended to be a rating of perceived exertion (RPE) of about 11-13 (coefficient of variation: 0.6). During the intervention, blood pressure and heart rate were continuously assessed using a monitor, and the intervention was implemented with careful attention to the safety of the patients, including any pain, hypotension, or cramping. The intervention period included some limitations. If patients wanted to participate in another exercise class at a different location, e.g., attend a gymnastics class or sports club, they were required to inform us so we could exclude confounding factors. Permission was given for the subjects to go through self-directed training similar to the intervention content on days other than the once-weekly intervention days; the number of iterations was self-reported on a regular basis.

\subsection{Data analysis}

The physical strength measurement values were evaluated by a repeated-measures one-way analysis of variance for comparison of the $\mathrm{BS}, 3 \mathrm{M}$, and $12 \mathrm{M}$ 
values, and Bonferroni correction was used after ANOVA for subsequent testing. Blood pressure data were comparatively studied using a two-factor repeated measures analysis of variance for the BS, exercise (Ex) day, non-Ex day, and before/after HD, while biochemical data were similarly comparatively studied using a repeated-measures twofactor analysis of variance over the $12 \mathrm{M}$ period, and before/after HD (except for Alb). The one-way ANOVA test was used for the $12 \mathrm{M}$ comparisons. SPSS 21.0 for windows (SPSS Inc., Chicago, IL) was used for all analyses, with p-values $<0.05$ considered statistically significant.

\section{Results}

The study subjects included 3 men and 5 women with an average age of $68.6 \pm 5.9$ years (4 subjects were in their $60 \mathrm{~s}$ and 4 in their $70 \mathrm{~s}$ ), with a HD history of $3.09 \pm 1.8$ years, weight $55.6 \pm 6.3 \mathrm{Kg}$, height $156.5 \pm 6.5 \mathrm{~cm}$, and body mass index (BMI) $21.8 \pm 2.1 \mathrm{~kg} / \mathrm{m}^{2} ; 6$ subjects had diabetes and $5 \mathrm{had}$ hypertension (Table 2). These participants started HD treatment at an elderly age. All subjects were able to continue with their exercises over the span of 1 year, with 3 subjects regularly implementing a home exercise program once a week or more, in a self-directed manner, during the time period.

The main outcome measure (physical function) was analyzed at 3 periods (BS, $3 \mathrm{M}$, and $12 \mathrm{M}$ ) by one-way analysis of variance. Statistically significant differences were observed for ankle plantar flexor strength, TUG, and maximum walking speed $(p<0.05)$ (Table 3). In the subsequent Bonferroni correction for these 3 items, the maximum walking speed showed no significant difference between the $\mathrm{BS}, 3 \mathrm{M}$, and $12 \mathrm{M}$, whereas significant differences between the BS and $3 \mathrm{M}$ were observed for TUG and ankle plantar flexion $(p<0.05)$. For body function items with a significant difference, the improvement rate by generation and sex was calculated. Regarding the percentage improvement rate by generation, we found improvements in the following items: BS and $3 \mathrm{M}(60 \mathrm{~s}: 19.4 \pm 18.3 \%, \mathrm{CV}: 1.1 ; 70$ s: $13.4 \pm 13.3 \%, \mathrm{CV}: 1.4)$, BS and $12 \mathrm{M}$ (60 s: 20.5 $\pm 23.0 \%, \mathrm{CV}: 1.6 ; 70 \mathrm{~s}: 15.4 \pm 16.0 \%, \mathrm{CV}: 1.7)$, and $3 \mathrm{M}$ and $12 \mathrm{M}(60 \mathrm{~s}: 4.6 \pm 14.8 \%$, CV 9.6; $70 \mathrm{~s}$ : $3.9 \pm 8.5 \%, \mathrm{CV}: 6.5$ ); regarding the improvement ratio by sex, BS and $3 \mathrm{M}$ (men: $19.2 \pm 10.0 \%, \mathrm{CV}$ : 0.8 ; women: $14.7 \pm 18.6 \%, \mathrm{CV}: 2.1$ ), BS and $12 \mathrm{M}$ (men: $15.3 \pm 22.1 \%$, CV: 5.6; women: $22.5 \pm 11.0$ $\%, C V: 0.8$ ), and $3 \mathrm{M}$ and $12 \mathrm{M}$ (men: $4.0 \pm 13.4 \%$, CV: 6.6; women: $4.7 \pm 3.5 \%, \mathrm{CV}: 1.0)$.

Regarding blood pressure data (Table 4), a paired two-factor analysis of variance was performed, using before/after HD and during the intervention period as independent variables and the measurement values as dependent variables, in order to verify whether differences would be observed in the measured values from before/after HD and during the intervention period. The main effects of the intervention period on blood pressure were not significant $(p>0.05)$, as well as for SBP or DBP, while the main effects of before/after HD were significant $(p<0.05)$. Moreover, the interactions were also not significant $(p>0.05)$. In terms of the heart rate, the main effects of the intervention period were not significant $(p>0.05)$, as well as the main effects of before/after HD and their interaction.

Similar to that performed for the blood pressure data, a paired two-factor analysis of variance was performed using before/after HD and during the intervention period as independent variables and the measurement values as dependent variables, for the biochemical data (Table 5). The main effects of before/after HD were significant for all biochemical

Table 2 Patient characteristics.

\begin{tabular}{llccccccc}
\hline Sex & $\begin{array}{c}\text { Age } \\
\text { (years) }\end{array}$ & $\begin{array}{c}\text { HD history } \\
\text { (years) }\end{array}$ & $\begin{array}{c}\text { Height } \\
(\mathrm{cm})\end{array}$ & $\begin{array}{c}\text { Weight } \\
(\mathrm{Kg})\end{array}$ & $\begin{array}{c}\text { BMI } \\
\left(\mathrm{Kg} / \mathrm{m}^{2}\right)\end{array}$ & DM & Hypertension \\
\hline 1 & MALE & 71 & 7.0 & 164 & 63.3 & 21.6 & - & - \\
2 & FEMALE & 71 & 4.0 & 148 & 59.5 & 24.0 & + & - \\
3 & FEMALE & 60 & 0.9 & 153 & 47.5 & 19.6 & + & + \\
4 & MALE & 71 & 3.3 & 165 & 57.0 & 21.5 & - & + \\
5 & FEMALE & 78 & 2.3 & 152 & 47.6 & 25.7 & + & + \\
6 & FEMALE & 65 & 0.8 & 152 & 51.7 & 21.4 & + & + \\
7 & MALE & 66 & 3.7 & 163 & 55.4 & 20.2 & + & + \\
8 & FEMALE & 62 & 3.3 & 155 & 63.2 & 20.0 & + & + \\
\hline
\end{tabular}


Table 3 Comparison of physical function before and after the intervention.

\begin{tabular}{|c|c|c|c|c|c|}
\hline & Baseline & $3 \mathrm{M}$ & $12 \mathrm{M}$ & $p$ & $\begin{array}{c}\text { Multiple } \\
\text { comparison }\end{array}$ \\
\hline \multicolumn{6}{|l|}{ Hand grip strength } \\
\hline Right (kg) & $19.4 \pm 5.6$ & $20.9 \pm 6.3$ & $20.8 \pm 7.3$ & 0.21 & \\
\hline Left $(\mathrm{kg})$ & $17.6 \pm 4.3$ & $18.4 \pm 4.5$ & $18.1 \pm 4.7$ & 0.44 & \\
\hline \multicolumn{6}{|l|}{ Leg muscle strength } \\
\hline Hip joint flex (Nm) & $23.6 \pm 11.5$ & $24.2 \pm 11.2$ & $27.5 \pm 10.1$ & 0.08 & \\
\hline Knee extensor (Nm) & $39.7 \pm 15.3$ & $41.3 \pm 15.0$ & $41.4 \pm 15.4$ & 0.32 & \\
\hline Ankle joint p/f (Nm) & $30.1 \pm 10.4$ & $34.8 \pm 5.2$ & $33.7 \pm 9.7$ & 0.03 & $\mathrm{BS}<3 \mathrm{M}$ \\
\hline Ankle joint $\mathrm{d} / \mathrm{f}(\mathrm{Nm})$ & $23.8 \pm 5.8$ & $27.8 \pm 7.3$ & $26.9 \pm 9.4$ & 0.21 & \\
\hline \multicolumn{6}{|l|}{ Walking speed } \\
\hline $10-\mathrm{m}$ normal $(\mathrm{Sec})$ & $8.1 \pm 2.0$ & $6.9 \pm 1.8$ & $6.8 \pm 2.2$ & 0.19 & \\
\hline 10-m maximum (Sec) & $9.8 \pm 2.4$ & $8.2 \pm 2.0$ & $8.6 \pm 3.2$ & 0.04 & n.s. \\
\hline TUG (Sec) & $10.1 \pm 2.9$ & $7.3 \pm 1.4$ & $7.8 \pm 2.6$ & $<0.00$ & $\mathrm{BS}>3 \mathrm{M}$ \\
\hline \multicolumn{6}{|l|}{ Static balance (eyes open) } \\
\hline LNG $(\mathrm{cm})$ & $76.5 \pm 19.1$ & $68.3 \pm 12.0$ & $68.4 \pm 10.4$ & 0.61 & \\
\hline $\mathrm{LNG} /$ time $(\mathrm{cm} / \mathrm{s})$ & $2.5 \pm 0.6$ & $2.3 \pm 0.4$ & $2.3 \pm 0.3$ & 0.61 & \\
\hline LNG/E.AREA $(1 / \mathrm{cm})$ & $20.7 \pm 3.7$ & $14.5 \pm 1.1$ & $14.6 \pm 2.0$ & 0.15 & \\
\hline \multicolumn{6}{|l|}{ Static balance (eyes closed) } \\
\hline LNG $(\mathrm{cm})$ & $99.3 \pm 15.9$ & $95.2 \pm 16.8$ & $97.2 \pm 16.8$ & 0.83 & \\
\hline $\mathrm{LNG} /$ time $(\mathrm{cm} / \mathrm{s})$ & $3.3 \pm 0.5$ & $3.2 \pm 0.6$ & $3.2 \pm 0.6$ & 0.83 & \\
\hline LNG/E.AREA $(1 / \mathrm{cm})$ & $17.1 \pm 3.5$ & $17.7 \pm 3.1$ & $15.6 \pm 2.9$ & 0.51 & \\
\hline
\end{tabular}

The data are presented as the mean \pm standard deviation. Abbreviations: M, months; BS, baseline; TUG, Timed Up and Go; LNG, length of body sway; E.AREA, sway excursion area; n.s., non-significant.

Table 4 Blood pressures and heart rates of the study patients.

\begin{tabular}{|c|c|c|c|c|c|c|c|c|c|}
\hline & \multicolumn{2}{|c|}{ Baseline } & \multicolumn{2}{|c|}{ Non-Ex day } & \multicolumn{2}{|c|}{ Ex day } & \multirow{2}{*}{$\begin{array}{c}p \\
\text { (HD Pre } \\
\text { vs. Post) }\end{array}$} & \multirow{2}{*}{$\begin{array}{c}p \\
\text { (Effect } \\
\text { of Ex) }\end{array}$} & \multirow{2}{*}{$\begin{array}{c}p \\
\text { (Interaction) }\end{array}$} \\
\hline & Pre HD & Post HD & Pre HD & Post HD & Pre HD & Post HD & & & \\
\hline $\mathrm{SBI}$ & $162.2 \pm 17.5$ & 136.7 & $165.1 \pm 19.1$ & 137.6 & 166.0 & 137 & $0.001 *$ & 0.83 & 0 . \\
\hline $\mathrm{DBP}$ & $82.9 \pm 6.1$ & $71.1 \pm 6.0$ & $80.6 \pm 7.4$ & $70.1 \pm 9.4$ & $81.4 \pm 6.4$ & $69.5 \pm 8.5$ & $0.001^{*}$ & 0.55 & 0.55 \\
\hline HR (bpm) & $75.1 \pm 8.8$ & $72.0 \pm 10.7$ & $73.0 \pm 11.4$ & $69.5 \pm 11.5$ & $69.4 \pm 15.1$ & $67.0 \pm 11.2$ & 0.63 & 0.14 & 0.81 \\
\hline
\end{tabular}

The data are presented as the mean \pm standard deviation. ${ }^{*}: p<0.01$.

Abbreviations: HD, hemodialysis; Ex, exercise; SBP, systolic blood pressure; DBP, diastolic blood pressure; HR, heart rate.

data $(p<0.05)$. However, the main effects of the intervention period were not significant $(p>0.05)$. Similarly, the interactions (before/after HD and during the intervention period) were also not significant $(p>0.05)$. Finally, there was no significant difference in the mean Alb level over the 12-month period $(p>0.05)$.

\section{Discussion}

In the present study, RT was implemented during $\mathrm{HD}$, and the intervention effects at $3 \mathrm{M}$ and $12 \mathrm{M}$ were clarified. Individualized exercise therapy comprising RT during HD was successfully implemented continuously for 1 year without dropouts. In terms of the physical function items, which were the main outcome measures, significant differences were observed between the BS and $3 \mathrm{M}$ in ankle plantar flexor strength, TUG, and maximum walking speed. However, no significant differences after intervention were observed in the secondary outcomes (blood pressure fluctuation and biochemical data).

From BS to $3 \mathrm{M}$, ankle plantar muscle strength and TUG were markedly improved. In patients with diabetic neuropathy, muscle atrophy occurs strongly among peripheral muscles (Johansen, et al, 2006). Furthermore, it is known that muscular strength decreases to a greater extent in the ankle joint muscle groups. Therefore, the effect on the ankle plantar muscles occurs more immediately than in the other muscle groups. In addition, because the strengthening of the plantar muscles was 


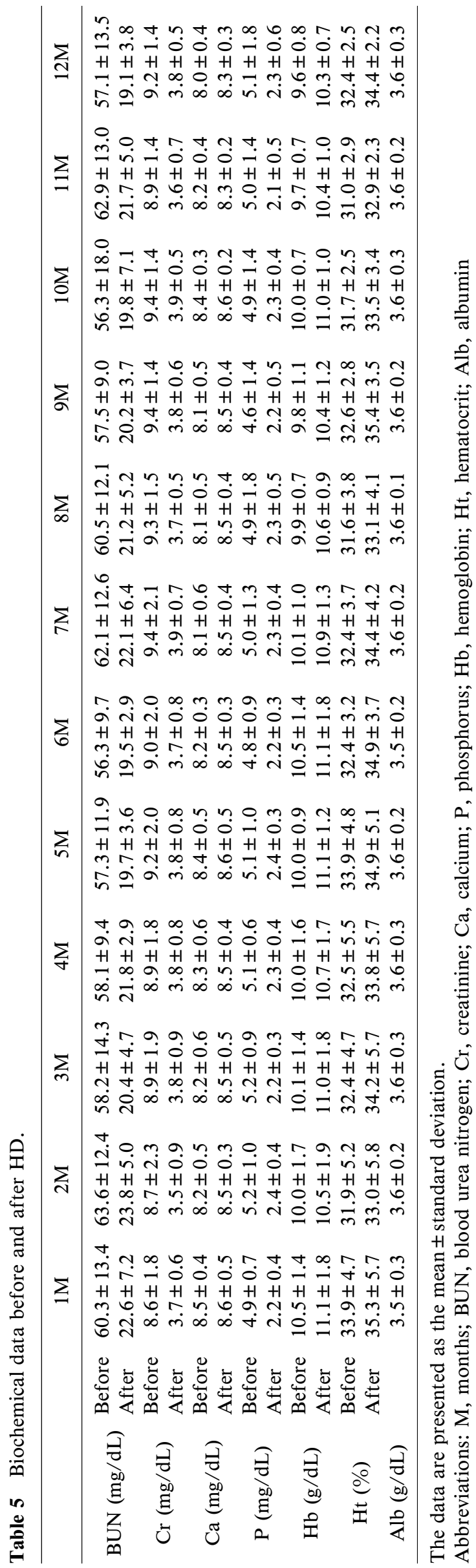

recognized, an improvement in TUG was seen, which is an indicator of the functional motion capability.

No changes were observed in most items, even after 12 months of intervention continuation. This suggests the possibility that, in addition to the intervention frequency likely being too low (once weekly), the exercise load, which was conducted at a lowto-medium intensity (11-13 RPE) and was mainly conducted in bed, may not have been enough; antigravity muscle training may be insufficient for maintaining physical function in elderly HD patients. Moreover, based on the result of the author's previous research, it can be speculated that these results may have been due to improvements of physical function at the initial stage (Shiota, et al., 2009; Shiota, 2011). In addition, as reported by Johansen et al. (2006), HD patients reportedly have a greater extent of muscle atrophy than inactive healthy subjects; however, according to other studies (Cheema B, et al., 2007), no improvement in muscle quality in these patients was observed. According to Roubenoff (2007), maintaining high physical activity every day and increasing muscle activity are regarded as important not only for muscle strength, but also for maintaining muscle mass. The activity level of the subjects in the present study was low; thus, intervention in the form of a program that adds to the muscle strength training during HD and accelerates the improvement of the physical activity for ADL is warranted. In the present study, no detailed exercises for improving physical activities in the patients' daily life, which have been shown in recent studies to be an important element to increase the daily physical activity, were provided. Improved effects on physical activity are expected if the patients can be motivated to exercise by themselves. Therefore, the RT exercise load, frequency, and duration require further study. Many dialysis patients have a negative perception of exercise and do not have exercise habits. Thus, noncompliance with exercise instructions is frequently encountered, which is problematic. This study had an advantage in that personal guidance could be conducted according to individual needs. In particular, we used RPE during resistance training, which allows for adaptation to these individual needs. In addition, as mentioned, unlike most previous studies, the present study examined the effects of low-tomedium intensity exercises in bed, and the subjects 
were elderly HD patients with wide-ranging ADL levels. These are some of the main advantages of this study.

Moreover, intervening with exercise therapy during HD eliminated dropouts, and blood pressure, pulse rate, and other vitals, including both objective and subjective symptoms, were confirmed on monitors by the healthcare provider during the intervention. As a result, during this 1-year intervention, no adverse events were observed. Nevertheless, there were also some limitations of the present study, including the lack of a control group and a small sample size. Therefore, there are items that are presumed to have a large standard deviation or no significant difference. In this study, we could not establish differences among sexes and differences by age because of the small sample size. Therefore, we can only make conclusions on a case-by-case basis regarding the intensity, frequency, and duration of exercise therapy. However, we show the importance of considering these factors when implementing RT. Therefore, for conducting RT during HD, the suitable exercise load and frequency first need to be established, and more effective exercise programs should be developed.

In conclusion, the present study was conducted to evaluate the effectiveness of low-to-medium intensity RT on physical function in elderly patients undergoing HD, after 1 year of once-weekly interventions. The results showed that RT during HD treatment had significant effects on ankle plantar flexor muscle strength, TUG, and maximum walking speed between the BS and after 3 months of intervention. However, because the RT conducted in the present study was of low-to-medium intensity, no significant differences were observed from $3 \mathrm{M}$ to 12 M. Nevertheless, subjects with a wide range of physical fitness levels could successfully participate in the study with no dropouts. This suggests that RT may represent an effective training method for elderly HD patients once its role and optimal frequency and intensity have been clearly defined.

\section{Acknowledgements}

I am sincerely grateful for the cooperation from Hiroshi HIROBE (ME), Makiko KOBAYASHI (ME), Toshihiko HASHIMOTO (MD), Yasuhiro IGUCHI (MD), and other staff of the Shinsui Dialysis Clinic, as well as the patients. I would also like to thank Editage for editing my manuscript.

\section{References}

Akima, H., Kubo, K., Imai, M., Kanehisa, H., Suzuki, Y., Gunji, A., and Fukunaga, T. (2001). Inactivity and muscle: effect of resistance training during bed rest on muscle size in the lower limb. Acta Physiol. Scand., 172: 269-278.

Bennett, P. N., Breugelmans. L., Barnard, R., Agius, M., Chan, D., Fraser, D., McNeill, L., and Potter, L. (2010). Sustaining a Hemodialysis Exercise Program: Rev. Semin. Dial., 23: 62-73.

Cheema, B., Abas, H., Smith, B., O’Sullivan, A., Chan, M., Patwardhan, A., Kelly, J., Gillin, A., Pang, G., Lloyd, B., and Singh, M.F. (2007). Progressive exercise for anabolism in kidney disease (PEAK): A randomized, controlled trial of resistance training during hemodialysis. J. Am. Soc. Nephrol., 18: 1594-1601.

Deligiannis, A., Kouidi, E., and Tourkantonis, A. (1999). Effects of physical training on heart rate variability in patients on hemodialysis. Am. J. Cardiol., 84: 197-202.

Heiwe, S. and Jacobson, S. H. (2014). Exercise Training in Adults With CKD: A Systematic Review and Meta-analysis. Am. J. Kidney Dis., 64: 383-393.

Johansen, K. L., Shubert, T., Doyle, J., Soher, B., Sakkas, G. K., and Kent-Braun, J. A. (2003). Muscle atrophy in patients receiving hemodialysis: Effects on muscle strength, muscle quality, and physical function. Kidney Int., 63: 291-297.

Johansen, K. L., Painter, P. L., Sakkas, G. K., Gordon, P., Doyle, J., and Shubert, T. (2006). Effects of resistance exercise training and nandrolone decanoate on body composition and muscle function among patients who receive hemodialysis: A randomized, controlled trial. J. Am. Soc. Nephrol., 17: 2307-2314.

Johansen, K. L. and Painter, P. (2012). Exercise in Individuals With CKD. Am. J. Kidney Dis., 59: 126-134.

Kouidi, E. (2002). Exercise training in dialysis patients: Why, when, and how?. Artif. Organs, 26: 1009-1013.

Longenecker, J. C., Coresh, J., Powe, N. R., Levey, A. S., Fink, N. E., Martin, A., and Klag, M. J.(2002). Traditional cardiovascular disease risk factors in dialysis patients compared with the general population: The CHOICE study. J. Am. Soc. Nephrol., 13: 1918-1927.

Mihaescu, A., Avram, C., Bob, F., Gaita D., Schiller, O., and Schiller, A. (2013). Benefits of Exercise Training during Hemodialysis Sessions: A Prospective Cohort Study. Nephron Clin. Pract., 124: 72-78.

Miller, B. W., Cress, C. L., Johnson, M. E., Nichols D. H., and Schnitzler, M. A. (2002). Exercise during hemodialysis decreases the use of antihypertensive medications. Am. J. Kidney Dis., 39: 828-833.

Moinuddin, I. and Leehey, D. J. (2008). A comparison of aerobic exercise and resistance training in patients with and without chronic kidney disease. Adv. Chronic Kidney Dis., 15: 83-96.

O'Hare, AM., Tawney, K., Bacchetti, P., and Johansen, K. L. (2003). Decreased survival among sedentary patients undergoing dialysis: Results from the dialysis morbidity and mortality study wave 2 . Am. J. Kidney Dis., 41: 447-454.

Parsons, T. L., Toffelmire, E. B., and King-VanVlack, C. E. (2004). The effect of an exercise program during hemodialysis on dialysis, efficacy, blood pressure and quality of life in endstage renal disease (ESRD) patients. Clin. Nephrol., 61: 261-274.

Pechter, Ü., Raag, M., and Ots-Rosenberg, M. (2014). Regular aquatic exercise for chronic kidney disease patients: a 10-year 
follow-up study. Int. J. Rehabil. Res., 37: 251-255.

Podsiadlo, D. and Richardson, S. (1991). The Timed "Up \& Go": A Test of Basic Functional Mobility for Frail Elderly Persons. J. Am. Geriatr. Soc., 39: 142-148.

Reid, R. D., Tulloch, H. E., Sigal, R. J., Kenny, G. P., Fortier, M., McDonnell, L., Wells, GA., Boulé, N. G., Phillips, P., and Coyle, D. (2010). Effects of aerobic exercise, resistance exercise or both, on patient-reported health status and well-being in type 2 diabetes mellitus a randomised trial. Diabetologia., 53: 632-640.

Maycon D. M, R., Diane. M. N, H., Ruiter. D. S, F., Alfredo. C. M. G, B., and Rogério. B. D, P. (2010). Exercise Training During Hemodialysis Reduces Blood Pressure and Increases Physical Functioning and Quality of Life. Artif. Organs, 34: 586-593.

Roubenoff, R. (2007). Physical activity, inflammation, and muscle loss. Nutr. Rev., 65: S208-S212.

Shiota, K., Takanashi, A., Ikeda, M., Oosawa, M., Suzuki, Y., and Hashimoto, T. (2009). Association between physical function and activities of daily living (ADL)/quality of life (QOL) in elderly dialysis patients. $5^{\text {th }}$ International Society Physical and Rehabilitation Medicine., 113-115.

Shiota, K., Hashimoto, T., Matsuda, T., Takanashi, A., and Ikeda, M. (2011). The Influence of a Physical Exercise during Hemodialysis in Improving Body Function. $6^{\text {th }}$ International Society Physical and Rehabilitation Medicine., 33-34.

Snowling, N. J. and Hopkins, W. G. (2006). Effects of different modes of exercise training on glucose control and risk factors for complications in type 2 diabetic patients-A meta-analysis. Diabetes Care., 29: 2518-2527.

Vilsteren, M. C., de Greef, M. H., and Huisman, R. M. (2005). The effects of a low-to-moderate intensity pre-conditioning exercise programme linked with exercise counselling for sedentary haemodialysis patients in The Netherlands: results of a randomized clinical trial. Nephrol. Dial. Transplant., 20: $141-146$.

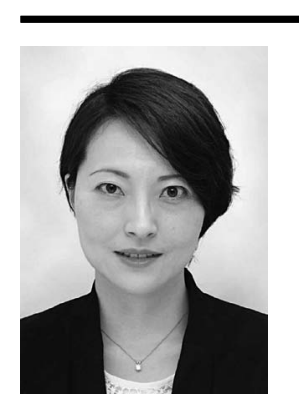

Name:

Kotomi Shiota

\section{Affiliation:}

Organization for University Research Initiatives, Waseda University

Institute of Health and Community Sports

Address:

Floor 3, Bldg. No. 9 Waseda Campus, Waseda University, 1-6-1 Nishiwaseda, Shinjuku-ku, Tokyo, 169-8050 JAPAN

Brief Biographical History:

-2016-Present Organization for University Research Initiatives, Waseda University

-2016- Present Institute of Health and Community Sports

-2011-2016 Waseda University

-Faculty of Sport Sciences-Assistant professor

-2007-2011 Ryotokuji University -Faculty of Health Sciences- Assistant professor

•2005-2009 Tokyo Metropolitan University - Doctoral Degree (Health Science)

-Health Science Research Department

-2003-2005 Tokyo Metropolitan University of Health Science - (Masters Degree)- Health Science Research Department Specialized in physical Therapy

\section{Main Works:}

-Shiota, K. (2016). Promotion and support of physical activity for elderly hemodialysis patients. J Phys Ther Sci, 28: 1378-1383.

-Shiota, K., Takanashi, A., Ikeda, M., Oosawa, M., Suzuki, Y., and Hashimoto, T. (2009). Association between physical function and activities of daily living (ADL)/quality of life (QOL) in elderly dialysis patients. $5^{\text {th }}$ International Society Physical and Rehabilitation Medicine., 113-115.

-Shiota, K. and Ikeda, M. (2008). Influence of aging change in visual system on postural control. J Jpn Health Sci, 11: 145-152.

Membership in Learned Societies:

-Japan Society of Physical Education, Health and Sport Sciences

- Japanese Physical Therapy Association

-International Society of Physical and Rehabilitation Medicine

- Japan Academy of Health Sciences 